\title{
Análisis de ADN fecal para identificar especies de felinos en los Ocotones Chiapas, México
}

\section{Fecal DNA analysis to identify feline species in Los Ocotones, Chiapas, Mexico}

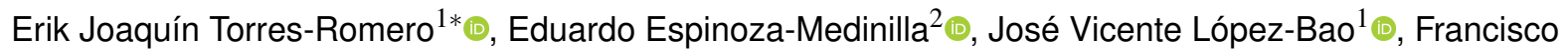 \\ Palomares ${ }^{3}$ (D) \\ ${ }^{1}$ Unidad de Investigación de Biodiversidad (UO/CSIC/PA), Universidad de Oviedo, 33600 Mieres, España. \\ ${ }^{2}$ Universidad de Ciencias y Artes de Chiapas, Libramiento Norte Poniente s/n, CP. 29039, Tuxtla Gutiérrez, Chiapas, México. \\ ${ }^{3}$ Estación Biológica de Doñana, CSIC. Departamento de Biología de la Conservación. Américo Vespucio s/n, CP. 41092, Isla de la \\ Cartuja, Sevilla, España. \\ *Autor de correspondencia: ejtr23@hotmail.com
}

Nota científica recibida: 24 de mayo de 2018 aceptada: 01 de octubre de 2018

RESUMEN. El conocimiento de las especies que se distribuyen en zonas con manejo forestal es fundamental para comprender los impactos negativos y positivos de esta actividad en la conservación de especies consideradas en protección por el gobierno mexicano a través de la NOM-059-SEMARNAT-2010, como es el caso del jaguar (Panthera onca). El objetivo fue identificar especies y sexo de felinos simpátricos presentes en el área de aprovechamiento forestal Los Ocotones (LOSOCO), ubicada a $36 \mathrm{~km}$ de Cintalapa, Chiapas. Se colectaron 33 muestras fecales y se analizaron para identificar perfiles genéticos individuales. Se identificaron dos pumas machos, un puma hembra, un jaguar macho, un ocelote/tigrillo hembra y un puma/leoncillo macho. El análisis genético aportó información relevante sobre el papel que juega la zona por albergar especies de felinos prioritarias para su conservación. Se requiere fortalecer las acciones de protección que ayuden a garantizar su permanencia en la región.

Palabras clave: Actividades humanas, corredor biológico, jaguar, leoncillo, ocelote, puma, tigrillo.

ABSTRACT. Knowledge of species that are distributed in areas with forest management is essential to understand the negative and positive impacts of this activity on the conservation of species considered for protection by the Mexican government through NOM-059-SEMARNAT-2010, such as the jaguar (Panthera onca). The objective was to identify the species and sex of sympatric felines present in the Los Ocotones forest use area (LOSOCO), located $36 \mathrm{~km}$ from Cintalapa, Chiapas. Thirty-three fecal samples were collected and analyzed to identify individual genetic profiles. Two male pumas, a female puma, a male jaguar, a female ocelot/tigrillo and a male puma/jaguarundi were identified. The genetic analysis provided relevant information on the role played by the area in hosting priority feline species for conservation purposes. It is necessary to strengthen protection actions that help ensure their permanence in the region.

Key words: Human activities, biological corridor, jaguar, jaguarundi, ocelot, puma, tigrillo.

\section{INTRODUCCIÓN}

Las presiones antrópicas en el medio ambiente han aumentado en todo el mundo en las últimas décadas, considerándose como un de los principales motores de pérdida de la biodiversidad (Ceballos et al. 2015). Los carnívoros, en particular los grandes felinos, se han visto afectados por la destrucción paulatina de sus ecosistemas, además de que, la caza y comercio ilegal han provocado que varias especies esten en peligro de extinción y muchas otras desapareciendo (Ripple et al. 2014, 2016). Sin embargo, estas especies al tener un amplio atractivo carismático y un perfil de visibilidad icónico más alto que otras, suelen ser elementos desencadenantes de conservación de la biodiversidad (Walpole y LeaderWilliams 2002, Ripple et al. 2014). También, ejercen o facilitan interacciones mediante el mantenimiento de cascadas tróficas controlando especies presa, y promoviendo un equilibrio en el ecosistema al alimen- 
tarse en ocasiones de cadáveres de animales que pueden albergar y propagar enfermedades (Ripple et al. 2014), además de que tienen un importante significado cultural (Treves y Bruskotter 2014).

En México, un total de 20 especies de mamíferos carnívoros terrestres y marinos presentan problemas de conservación, y se encuentran en alguna categoría de protección especial en la NOM-059-SEMARNAT-2010 (SEMARNAT 2010). De manera particular, el estado de Chiapas aún mantiene hábitats adecuados para muchas especies de plantas y animales prioritarias para la conservación, registrándose cinco de las seis especies de felinos silvestres (jaguar, Panthera onca; leoncillo, Puma yagouaroundi; puma, Puma concolor; ocelote, Leopardus pardalis y tigrillo, Leopardus wiedii), que se distribuyen en México (Ceballos et al. 2010, Espinoza-Medinilla et al. 2018). Pero los hábitats naturales que aún persisten están siendo afectados por la destrucción y fragmentación de actividades humanas, lo que ocasiona mosaicos de vegetación con diferente grado de perturbación (Dirzo et al. 2009). Por ejemplo, la extracción de madera tiene impacto sobre los ecosistemas, por el establecimiento de máquinas y vehículos, presencia constante de trabajadores, construcción y mantenimiento de caminos y ruido; los cuales afectan los servicios ambientales y la biodiversidad (Rendón et al. 2014). En este sentido, el aprovechamiento forestal podría afectar de manera directa a los felinos, ya que especies como el jaguar, leoncillo y tigrillo, suelen presentar menos tolerancia a las presiones humanas y tienden a desplazarse a otras áreas; y en última instancia desaparecer a nivel local o regional (Ceballos et al. 2010, De Angelo et al. 2011, Giordano 2016). Pero hay predios privados como Los Ocotones (LOSOCO) que promueven un manejo forestal sustentable por mantener vegetación nativa para proteger la cobertura arbórea alta, además de ser hábitat que permite la conexión con otros remanentes de vegetación de importancia biológica y ecológica. El predio LOSOCO mantiene vegetación natural para refugio o sitios de reproducción y alimentación de especies de felinos. Además de favorecer la movilidad y dispersión de felinos para otros remanentes de vegetación y la re- colonización de otras áreas (Espinoza-Medinilla et al. 2018).

Los felinos son especies cripticas, nocturnas y de difícil visualización (Torres-Romero et al. 2017), por lo que realizar estudios con técnicas convencionales como la manipulación directa para la marcación o radio-telemetría, son logísticamente difíciles y los costos suelen ser elevados (Ruell y Crooks 2007). En contraste, las técnicas indirectas, como el foto-trampeo y el rastreo permiten a los investigadores conocer aspectos ecológicos y/o biológicos de las especies de manera no invasiva, pero en ocasiones la información resultante no permite diferenciar entre especies y sexo (Anderson et al. 2003, Ruell y Crooks 2007). Por lo anterior, el análisis genético de muestras fecales puede utilizarse como método no invasivo que permite determinar características importantes como la especie, sexo, tamaño poblacional, variabilidad genética, filogeografía y relaciones filogenéticas; que pueden ser difíciles de determinar con otros métodos (Kurose et al. 2005, Naidu et al. 2012).

En este contexto, el conocimiento básico sobre los felinos silvestres en áreas con algún grado de perturbación humana es un paso fundamental para contribuir a diseñar estrategias de conservación de especies y de hábitats en ambientes fragmentados o modificados por actividades antrópicas. Por lo anterior el objetivo fue identificar especies y sexo de felinos simpátricos presentes en el área de aprovechamiento forestal Los Ocotones.

\section{MATERIALES Y MÉTODOS}

\section{Área de estudio}

El predio LOSOCO ( $16^{\circ} 38^{\prime} 59^{\prime \prime}$ LN y $93^{\circ} 47^{\prime}$ 59" LO) ocupa 2400 ha, se ubica a 36 km de Cintalapa, Chiapas, a $40 \mathrm{~km}$ al Sureste de la Reserva de la Biosfera La Sepultura y al Noreste de la Reserva El Ocote (Figura 1). El área es parte de un gran bloque de vegetación en buen estado de conservación conocido como Selva Zoque, abarca municipios de los estados de Veracruz, Oaxaca y Chiapas, además de incluir a la Sierra Atravesada en Oaxaca y la Sierra Madre de Chiapas (Lira-Torres et al. 2012). 


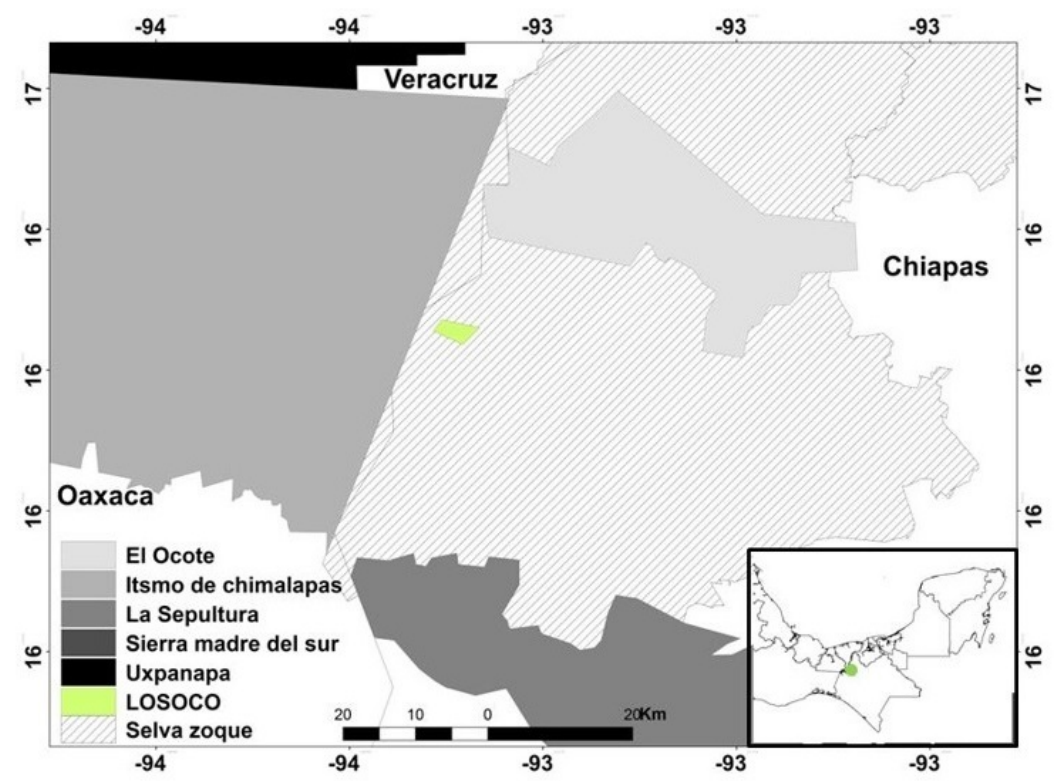

Figura 1. Ubicación de la unidad de manejo forestal Los Ocotones (LOSOCO), Chiapas, Selvas de importancia para la conservación y Áreas Naturales Protegidas, México.

No obstante, la Selva Zoque tiene problemas de fragmentación y cambio de uso del suelo. Sin embargo, es un área que puede considerarse como corredor biológico conectado con otras selvas como la Reserva de la Biosfera el Ocote y la Sepultura en Chiapas, los Chimalapas en Oaxaca y la región del Uxpanapa en Veracruz, reconocidas por su importancia para la conservación de la biodiversidad regional (CONANP 2015). LOSOCO está certificada por Rainforest Alliance y Forest Stewardship Council por su manejo forestal sostenible. Cuenta con un jardín botánico y tres unidades de manejo para la conservación de vida silvestre (UMAs) de venado cola blanca, cycadas y orquídeas. El clima es cálidohúmedo con temperatura anual de 18 a $22{ }^{\circ} \mathrm{C}$, mayo es el mes más cálido con $18{ }^{\circ} \mathrm{C}$ con precipitación anual de 1000 a $1800 \mathrm{~mm}$ (Arriaga et al. 2000). La altitud oscila entre 1000 y 1220 msnm (INEGI 2005). La vegetación de LOSOCO es diversa, pero predomina el bosque de pino-encino y el bosque tropical subcaducifolio (Rzedowski 1978); de ellos, el bosque de pino es el que más se aprovecha.

\section{Recolección y conservación de muestras}

La recolecta de las muestras fecales se realizó durante dos días en temporada seca en el mes de mayo del 2011. Los recorridos se realizaron a pie a lo largo de los principales caminos y veredas cubriendo toda el área de estudio (2 400 ha), iniciando la búsqueda de 06:00 a 18:00 h. De acuerdo con Aranda (2000) se recolectaron todas las excretas que estuvieran presentes durante el muestreo, considerando aquellas muestras que cumplieran los requisitos de color, olor, forma y tamaño, junto a huellas 0 restos de presas, presencia de pelos 0 fragmentos de huesos. Las muestras recolectadas se georreferenciaron con GPS, las muestras frescas se almacenaron con etanol al $96 \%$ de 24 a $48 \mathrm{~h}$ y después se transfirieron a gel de sílice para su almacenamiento. Mientras que las muestras secas se almacenaron en envases esterilizados y herméticos de plástico de $100 \mathrm{ml}$ con gel de sílice, permaneciendo de esta forma hasta su análisis genético.

\section{Extracción de ADN para la identificación de es-} pecies y sexado

La identificación de especies por medio 
de excretas se realizó mediante marcadores moleculares específicos para felinos desarrollados por Roques et al. (2011). La extracción de ADN de heces se realizó de acuerdo con el protocolo basado en el método GuSCN/sílice (Höss y Paabo 1993, Frantz et al. 2003). Para controlar la contaminación, durante la extracción del ADN y PCR, el procedimiento se realizó en un laboratorio diseñado para manipular material no invasivo. Para la identificación, se utilizaron los siguientes oligonucleótidos (primers): ND5-FEL-274R, ND5-FEL-130F, ND5-Po-149F, ND5-Hy-58F, ND5-Pc/Hy-183F, y ND5$\mathrm{Fp} / \mathrm{Fw}$-167F, específicos para felinos neotropicales (Tabla 1). La identificación sexual se realizó con el método descrito por Pilgrim et al. (2005) y optimizado para su uso en muestras fecales de felinos (Puma concolor, Leopardus pardalis, Leopardus wiedii) por Roques et al. (2011) y (2014).

\section{RESULTADOS Y DISCUSIÓN}

Se recolectaron y analizaron 33 muestras fecales en el área de estudio. Del total de muestras fecales, $22(66.66 \%)$ fueron de $P$. concolor, $2(6.09 \%)$ de $P$. onca, $3(9.09 \%)$ de $L$ pardalis/L. wiedii, 3 (9.09) de $P$. concolor/P. yaguaroundi y $3(9.09 \%)$ no amplificaron. Para la identificación a nivel de sexo, se identificaron dos individuos machos y una hembra de $P$. concolor, un macho $P$. onca, un L. pardalis/L. wiedii hembra y un $P$. concolor/P. yaguaroundi macho (Tabla 2). Las muestras en las que no se pudo determinar la especie y sexo fue por la baja calidad y cantidad de ADN. No obstante, la tasa de éxito para la identificación a nivel de especie fue del $72.72 \%$, mientras que el sexo fue al $78.78 \%$.

Los felinos son considerados especies indicadoras del estado de la biodiversidad y de conservación de los ecosistemas naturales (Ordiz et al. 2013, Ripple et al. 2014). Además, especies icónicas como el jaguar han sido utilizadas como especie clave en iniciativas de conservación (Manterola et al. 2011), y para el diseño de corredores biológicos (Salom-Pérez et al. 2010, Hidalgo-Mihart et al. 2018). Bajo este contexto, y por las características de hábitat que presenta la zona de manejo forestal LOSOCO se identificaron especies de felinos relevantes, de las que el jaguar, el leoncillo, el ocelote y el tigrillo tienen estatus de especies en peligro de extinción y amenazadas; mientras que el puma, es una especie que requiere de protección especial en la NOM-059 (SEMARNAT 2010). Pese a las modificaciones causadas por el hombre por la extracción de madera dentro de los LOSOCO, la unidad de manejo mantiene su hábitat natural para albergar especies prioritarias para su conservación.

El hábitat que presenta LOSOCO, adquiere relevancia por albergar a especies prioritarias y por jugar un papel importante como corredor de dispersión para especies con requerimientos espaciales mayores como el jaguar y el puma. Al respecto, se ha reportado que el puma y el jaguar presentan promedios en sus ámbitos hogareños que oscilan entre $328.8 \pm 54.9 \mathrm{~km}^{2}$ (pumas machos) y 151.1 $\pm 26.4 \mathrm{~km}^{2}$ (pumas hembras), mientras que para el jaguar oscilan entre $203.3 \pm 132.8 \mathrm{~km}^{2}$ (jaguares machos) y $149 \pm 97.1 \mathrm{~km}^{2}$ (jaguares hembras) (González-Borrajo et al. 2017); por lo que su amplio rango de movimiento entre especies machos y hembras permite tener una gran movilidad entre parches conectados para mantener o aumentar las tasas de movimiento hacia otros remanentes de vegetación (Salom-Pérez et al. 2010). Además ofrece beneficios a otras especies con requerimientos espaciales menores como el ocelote, leoncillo y tigrillo (Ceballos et al. 2010, Ripple et al. 2014). Por lo que, los corredores deben ser estrategias indispensables para mantener las poblaciones genéticamente sanas mediante el intercambio de individuos (Frankham 2006). En consecuencia y teniendo en cuenta el ámbito hogareño promedio entre el puma y el jaguar que oscila entre $281.87 \pm 35.76 \mathrm{~km}^{2}$ y $128.61 \pm 49.5 \mathrm{~km}^{2}$, respectivamente (GonzálezBorrajo et al. 2017), se infiere de que el predio LOSOCO cuenta con vegetación natural para refugio, sitio de reproducción y de alimentación para especies de felinos, además de contar con características para ser sitio de movilidad y de dispersión hacia otras áreas con importantes remanentes de vegetación, como la Reserva de la Biosfera el Ocote y Sepultura en Chiapas, los Chimalapas en Oaxaca, y Uxpanapa 
Tabla 1. Oligonucleótidos (primers) usados en el estudio.

\begin{tabular}{llcc}
\hline Primers & Secuencia & $\mathrm{C}^{N}$ (in $\left.\mu \mathrm{M}\right)$ & PCR product \\
\hline ND5-FEL-274R & YGTAGCACTTTKYGTCACATGA & 0.25 & \\
ND5-FEL-130F & CCTTYACYATCAGCATAAT & 0.30 & $166 \mathrm{BPS}$ \\
ND5-Po-149F & TCCGGCTATAGTATTTATTTCTTCC & 0.25 & $147 \mathrm{BPS}$ \\
ND5-Py-58F & TCATTATAACCGGCACCCAACTG & 0.25 & $238 \mathrm{BPS}$ \\
ND5-Pc/Py-183F & GCAGTCATCTCAAACTGACACTG & 0.25 & $113 \mathrm{BPS}$ \\
ND5-Lp/Lw-167F & TYTCCCAGGACAAGAAGCAGTA & 0.20 & $129 \mathrm{BPS}$ \\
\hline FEL, las cinco especies objetivo; Po, Panthera onca; Py, Puma yagouaroundi; Pc, Puma \\
Concolor; Lp, Leopardus pardalis; Lw, Leopardus wiedii. C ${ }^{N}$, concentración del primer final \\
en PCR.
\end{tabular}

Tabla 2. Resultados de la amplificación genética en muestras fecales recolectadas en el campo.

\begin{tabular}{|c|c|c|c|}
\hline Identificación muestra fecal & Identificación de especie & Sexo & ID-individuo \\
\hline $8 \mathrm{~F} 190511$ & Jaguar & $\mathrm{MNI}$ & $\mathrm{MNI}$ \\
\hline 06C190511 & Jaguar & Macho & $\mathrm{J}-\mathrm{M}-1$ \\
\hline 1F190511 & Ocelote/Tigrillo & $\mathrm{MNI}$ & MNI \\
\hline $01 C 190511$ & Ocelote/Tigrillo & Hembra & \\
\hline 04C190511 & Ocelote/Tigrillo & Hembra & \\
\hline 10F190511 & Puma & Macho & P-M-1 \\
\hline 1F180511 & Puma & Macho & \\
\hline $2 F 180511$ & Puma & Macho & P-M-1 \\
\hline 2F190511 & Puma & Macho & $\mathrm{MNI}$ \\
\hline 3F180511 & Puma & Macho & P-M-1 \\
\hline 3F190511 & Puma & Macho & P-M-1 \\
\hline 4F190511 & Puma & Macho & P-M-2 \\
\hline $5 F 190511$ & Puma & Macho & P-M-1 \\
\hline 6F190511 & Puma & Macho & P-M-1 \\
\hline 7F190511 & Puma & $\mathrm{MNI}$ & $\mathrm{MNI}$ \\
\hline 9F190511 & Puma & Macho & P-M-1 \\
\hline $01 C 180511$ & Puma & Macho & P-M-1 \\
\hline $02 \mathrm{C} 180511$ & Puma & Hembra & \\
\hline 02C190511 & Puma & Hembra & $\mathrm{P}-\mathrm{H}-1$ \\
\hline $03 C 180511$ & Puma & Hembra & $\mathrm{P}-\mathrm{H}-1$ \\
\hline 03C190511 & Puma & Macho & P-M-1 \\
\hline 04C180511 & Puma/Leoncillo & Macho & \\
\hline $05 \mathrm{C} 180511$ & Puma & Hembra & $\mathrm{P}-\mathrm{H}-1$ \\
\hline 05C180511-B & Puma & Hembra & $\mathrm{P}-\mathrm{H}-1$ \\
\hline $05 C 190511$ & Puma & Macho & P-M-1 \\
\hline $06 C 180511$ & Puma & Hembra & $\mathrm{P}-\mathrm{H}-1$ \\
\hline 07C190511 & Puma & Macho & P-M-1 \\
\hline $08 C 180511$ & Puma/Leoncillo & Macho & \\
\hline $08 C 190511$ & Puma & Macho & P-M-1 \\
\hline $10 \mathrm{C} 180511$ & Puma/Leoncillo & MNI & $\mathrm{MNI}$ \\
\hline 4F180511 & $\mathrm{MNI}$ & $\mathrm{MNI}$ & $\mathrm{MNI}$ \\
\hline $07 C 180511$ & $\mathrm{MNI}$ & $\mathrm{MNI}$ & MNI \\
\hline $09 C 180511$ & $\mathrm{MNI}$ & $\mathrm{MNI}$ & MNI \\
\hline
\end{tabular}

en Veracruz. La importancia de LOSOCO como lugar de presencia permanente de especies como el jaguar y puma, y área de reproducción, debe ser evaluada. Por lo que es imprescindible continuar con estudios en la zona de estudio y las regiones adyacentes para identificar el papel de Los Ocotones en la conectividad de las especies a nivel regional.

\section{AGRADECIMIENTOS}

A M. Hidalgo-Mihart, a los dos revisores anónimos por sus comentarios constructivos al manuscrito. Al apoyo del Señor Ariel Gómez Jiménez propietario de Los Ocotones por las facilidades otorgadas para el desarrollo de la investigación. 


\section{LITERATURA CITADA}

Anderson DR, Cooch EG, Gutierrez RJ, Krebs CJ, Lindberg MA, Pollock KH, et al. (2003) Rigorous science: suggestions on how to raise the bar. Wildlife Society Bulletin 31: 296-305.

Aranda M (2000) Huellas y otros rastros de los mamíferos grandes y medianos de México. Instituto de Ecología, Conabio. México. 212p.

Arriaga L, Espinoza JM, Aguilar C, Martínez E, Gómez L, Loa E (2000) Regiones terrestres prioritarias de México. Comisión Nacional para el Conocimiento y Uso de la Biodiversidad. México. 605p.

Ceballos G, List R, Medellín R, Bonacic, C, Pacheco J (2010) Los felinos de América. Cazadores sorprendentes. México. 303p.

Ceballos G, Ehrlich PR, Barnosky AD, Garcia A, Pringle RM, Palmer TM (2015) Accelerated modern humaninduced species losses: entering the sixth mass extinction. Science Advances 1, 5, e1400253. Doi: 10.1126/sciadv. 1400253

CONANP (2015) Resolución sobre las cifras oficiales correspondientes a las superficies de las Áreas Naturales Protegidas de competencia Federal en México. México. 18p. http://www.conanp.gob.mx/. Fecha de consulta: 3 de abril de 2018.

De Angelo C, Paviolo A, Di Bitetti M (2011) Differential impact of landscape transformation on pumas (Puma concolor) and jaguars (Panthera onca) in the upper Paraná Atlantic Forest. Diversity and Distributions 17: $422-436$.

Dirzo R, Aguirre A, López JC (2009) Diversidad florística de las selvas húmedas en paisajes antropizados. Investigación Ambiental 1: 17-22.

Espinoza-Medinilla E, Torres-Romero EJ, Tarango-Arámbula LA (2018) Additional records of wild mammals in the forest management area: Los Ocotones, Chiapas, Mexico. Agrociencia 52: 553-562

Frantz AC, Pope LC, Carpenter PJ, Roper TJ, Wilson GJ, Delahay RJ, et al. (2003) Reliable microsatellite genotyping of the Eurasian badger (Meles meles) using faecal DNA. Molecular Ecology 12: 1649-1661.

Frankham R (2006) Genetics and landscape connectivity. In: Crooks KR, Sanjayan M (comps.). Connectivity conservation. Cambridge University Press. Cambridge, England. pp. 72-96.

Giordano AJ (2016) Ecology and status of the Jaguarundi Puma yagouaroundi: a synthesis of existing knowledge. Mammal Review 46: 30-43

González-Borrajo N, López-Bao JV, Palomares F (2017) Spatial ecology of jaguars, pumas, and ocelots: a review of the state of knowledge. Mammal Review 47: 62-75.

Hidalgo-Mihart M, Contreras-Moreno F, Jesús de la Cruz A, Juárez-López R (2018) Validation of the CalakmulLaguna de Terminos corridor for jaguars Panthera onca in south-eastern Mexico. Oryx 52: 292-299

Höss M, Paabo S (1993) DNA extraction from pleistocene bones by a silica- based purification method. Nucleic Acids Research 21: 3913-3914.

INEGI (2005) Prontuario de información geográfica municipal de los Estados Unidos Mexicanos. Cintalapa, México. Instituto Nacional de Estadística, Geografía e Informática. Mexico. 9p.

Kurose N, Masuda R, Tatara M (2005) Fecal DNA analysis for identifying species and sex of sympatric carnivores: a noninvasive method for conservation on the Tsushima islands, Japan. Journal of Heredity 96: 688-697. 
Lira-Torres I, Galindo C, Briones-Salas M (2012) Mamíferos de la Selva Zoque: riqueza, uso y conservación. Revista de Biología Tropical 60: 781-797

Manterola C, Conde DA, Colchero F, Rivera A, Huerta E, Soler A, et al. (2011) El Jaguar como elemento estratégico para la conservación. Comisión Nacional para el Conocimiento y Uso de la Biodiversidad. Corredor Biológico Mesoamericano México. Serie Acciones/Número 8 México. 126p

Naidu A, Fitak RR, Munguia-Vega A, Culver M (2012) Novel primers for complete mitochondrial cytochrome b gene sequencing in mammals. Molecular Ecology Resources 12: 191-196.

Ordiz A, Bischof R, Swenson JE (2013) Saving large carnivores, but losing the apex predator? Biological Conservation 168: 128-133

Pilgrim KL, McKelvey KS, Riddle AE, Schwartz MK (2005) Felid sex identification based on noninvasive genetic samples. Molecular Ecology Notes 5: 60-61.

Rendón H, Martínez A, Pérez DR (2014) Los bosques, sus bienes y servicios: los retos del manejo forestal sustentable. Ciencias 111-112: 29:35

Ripple WJ, Estes JA, Beschta RL, Wilmers CC, Ritchie EG, Hebblewhite M, et al. (2014) Status and ecological effects of the world's largest carnivores. Science 343: 151-163.

Ripple WJ, Chapron G, López-Bao JV, Durant SM, Macdonald DW, Lindsey PA, Corlett RT (2016) Saving the world's terrestrial megafauna. BioScience 66: 807-812.

Roques S, Adrados B, Chavez C, Keller C, Magnusson WE, Palomares F, et al. (2011) Identification of Neotropical felid faeces using RCP-PCR. Molecular Ecology Resources 11: 171-175.

Roques S, Furtado M, Jácomo ATA, Silveira L, Sollmann R, Torres NM, et al. (2014) Monitoring jaguar populations Panthera onca with non-invasive genetics: a pilot study in Brazilian ecosystems. Oryx 48: 361-369.

Ruell WW, Crooks KR (2007) Evaluation of non-invasive genetic sampling methods for felid and canid populations. Journal of Wildlife Management 71: 1690-1694.

Rzedowski J (1978) La vegetación de México. Edit. Limusa. México. 432p.

Salom-Peréz R, Polisar J, Quigley H, Zeller K (2010) Iniciativa del corredor del jaguar: Un corredor biológico y un Compromiso a largo plazo para la conservación. Revista Mesoamericana 14: 25-34.

SEMARNAT (2010) Norma Oficial Mexicana NOM-059- SEMARNAT-2010, Protección ambiental - Especies nativas de México de flora y fauna silvestres - Categorías de riesgo y especificaciones para su inclusión, exclusión o cambio - Lista de especies en riesgo. Diario Oficial de la Federación. 30 de diciembre de 2010, Segunda Sección. Ciudad de México.

Treves A, Bruskotter JT (2014) Tolerance for predatory wildlife. Science 344: 476-477.

Torres-Romero EJ, Espinoza-Medinilla E, Lazcano-Barrero MA, Maffei L (2017) Ecology and conservation of ocelot (Leopardus pardalis) in Northern Quintana Roo, Mexico. Therya 8: 11-18.

Walpole MJ, Leader-Williams N (2002) Tourism and flagship species in conservation. Biodiversity Conservation 11: 543-547. 
